\title{
Foreword
}

\section{Birth of JAD: 20 Years Later}

Twenty years ago, the editorship of the Journal of Alzheimer's Disease (JAD) was offered to George Perry by Einar Fredriksson, President of IOS Press, and his US publisher Yale Altman, after George had shared with him some thoughts on steps that could lead JAD to a preeminent place in the field of Alzheimer's disease within but a few years. Einar was prophetic in tempering George's enthusiasm with the steps and years necessary to achieve that goal. The plan was to design a journal that reflected the field through creating community. Editorial structure, openness to novel topics, reviewer selection, scientometric analysis, awards programs, and historical compendiums were all focused on building a JAD community.

Beth Kumar, our Managing Editor, was instrumental in providing the vision, innovation, organization-and most of all, tenacity to secure reviews - that provided the glue to hold all our initiatives together and serve as the steady force of JAD. Eileen Leahy, Marketing Coordinator (IOS Press), skillfully guided PubMed and ISI indexing, and press releases. Rasjel van der Holst, JAD Associate Publisher (IOS Press), brought a new professionalism to journal function, essential to a firm grounding. Analyses by Aaron Sorensen, our Scientometrics Editor, of Alzheimer's disease publication, citation, and funding trends has brought new resources and insight to enable the field to understand itself. And JAD would not be JAD without the constant bantering of Mark A. Smith to push the envelope of what a journal could add to our field. Finally, we thank the endless efforts of our Deputy, Regional, Ethics, Reviews, Senior, and Associate Editors and the thousands of reviewers who have assured JAD's quality.

In 20 years, JAD has published more papers on $\mathrm{AD}$ than any other journal and received more citations than any other AD-focused journal. JAD is now published semi-monthly, has a parallel book series, and has launched the open access JAD Reports, as well as sister journals on Parkinson's, Huntington's and Neuromuscular Diseases.

The JAD community of authors now celebrates the past 20 years by writing of the journal's role in developing the field, and in many cases their careers. It is a great honor to celebrate 20 years of community partnership.

George Perry Jesus Avila Massimo Tabaton Xiongwei Zhu 